Journal of the Operations Reserch

Society of Japan

Vol. 19, No. 2, June, 1976

\title{
AN ITERATION METHOD FOR NONLINEAR PROGRAMMING PROBLEMS
}

\author{
HISASHI MINE and KATSUHISA OHNO, Kyoto University \\ TATSUO NODA, Toyama College of Technology
}

(Received February 26, 1975)

\begin{abstract}
The purpose of this paper is to propose a simple and practical iteration method for solving a nonlinear programming problem. It can be shown that the sequence of points generated by the iteration method converges to a 1 local optimum solution of the nonlinear programming problem.
\end{abstract}

*This work was done while the third author was studying at Department of Applied Mathematics and Physics, Kyoto University as a visiting reseacher from October 1974 to March 1975. 
1. Introduction

Let $R^{n}$ be the $n$-dimensional Euclidean space, and let $h_{i}\left(x_{1}, x_{2}, \ldots, x_{n}\right)$ $(i=1,2, \ldots, m)$ and $f\left(x_{1}, x_{2}, \ldots, x_{n}\right)$ be nonlinear and real-valued functions defined on $R^{n}$. Consider the following nonlinear programming problem:

$$
\begin{aligned}
& \text { Minimize } f\left(x_{1}, x_{2}, \ldots, x_{n}\right) \\
& \text { subject to }\left(x_{1}, x_{2}, \ldots, x_{n}\right) \in D
\end{aligned}
$$

where

$$
D=\left\{\left(x_{1}, x_{2}, \ldots, x_{n}\right) ; h_{1}\left(x_{1}, x_{2}, \ldots, x_{n}\right) \leqq 0, i=1,2, \ldots, m\right\} .
$$

In general, it seems difficult to find the global minima of the problem (1). In some practical cases, however, local minima are no less important than global minima. In this paper, consequently, we deal with the problem of finding a local minimum $\left(\bar{x}_{1}, \bar{x}_{2}, \ldots, \bar{x}_{n}\right) \in D$ of nonlinear programming problem (1).

Let us introduce slack variables $x_{n+i}(i=1,2, \ldots, m)$ and define functions $g_{i}\left(x_{1}, x_{2}, \ldots, x_{n+m}\right)(i=1,2, \ldots, m)$ as

$$
g_{i}\left(x_{1}, x_{2}, \ldots, x_{n+m}\right)=h_{i}\left(x_{1}, x_{2}, \ldots, x_{n}\right)+x_{n+i} 2
$$

Then problem (1) can be rewritten as:

$$
\begin{aligned}
& \text { Minimize } f\left(x_{1}, x_{2}, \ldots, x_{n}\right) \\
& \text { subject to } \\
& g_{i}\left(x_{1}, x_{2}, \ldots, x_{n+m}\right)=0 \quad(i=1,2, \ldots, m), \\
& x_{j} \in R(j=1,2, \ldots, n+m) .
\end{aligned}
$$

First, Lagrangian function $\phi\left(x_{1}, x_{2}, \ldots, x_{n+m} ; \lambda_{1}, \lambda_{2}, \ldots, \lambda_{m}\right)$ associated with problem (2) is introduced as

$$
\phi=f\left(x_{1}, x_{2}, \ldots, x_{n}\right)+\sum_{i=1}^{m} \lambda_{i} g_{i}\left(x_{1}, x_{2}, \ldots, x_{n+m}\right) .
$$

Define an $(n+m)$-dimensional vector $x$, an $m$-dimensional vector $\lambda$ and an $m$-dimensional vector-valued function $g(x)$ as follows:

$$
\begin{aligned}
& x=\left(x_{1}, x_{2}, \ldots, x_{n+m}\right), \\
& \lambda=\left(\lambda_{1}, \lambda_{2}, \ldots, \lambda_{m}\right),
\end{aligned}
$$


and

$$
g(x)=\left(g_{1}(x), g_{2}(x), \ldots, g_{m}(x)\right) .
$$

Denote by * the transpose. Then, Lagrangian (3) is reduced to

$$
\phi(x, \lambda)=f(x)+\lambda(g(x))^{*} .
$$

2. The Main Theorem

It is assumed that $h_{i}(i=1,2, \ldots, m)$ and $f$ are three times continuously differentiable on $R^{n}$. Denote by $\partial g(x) / \partial x$ the $m \times(n+m)$ matrix with $(i, j)$ component $\partial g_{i}(x) / \partial x_{j}$. Define an $(n+2 m)$-dimensional vector $p(x, \lambda)$ and an $(n+2 m) x$ $(n+2 m)$ matrix $A(x, \lambda)$ as follows:

$$
p(x, \lambda)=\left(\phi_{x}(x, \lambda), \phi_{\lambda}(x, \lambda)\right),
$$

and

$$
A(x, \lambda)=\left(\begin{array}{cc}
\phi_{x x}(x, \lambda) & (\partial g(x) / \partial x)^{*} \\
\partial g(x) / \partial x & 0
\end{array}\right),
$$

where $\phi_{x}$ and $\phi_{\lambda}$ are gradient vectors with components $\partial \phi / \partial x_{j}$ and $\partial \phi / \partial \lambda_{i}$ respectively, and $\phi_{x x}$ is the Hessian matrix with $(j, k)$ component $\partial^{2} \phi / \partial x_{j} \partial x_{k}$. Then the following lemma is well known. (see, for example, Hadley [4, page 101]).

Lemma 1. Suppose that the following conditions (a)-(c) are satisfied:

(a) There exists at least one of solutions $(\bar{x}, \bar{\lambda}), \bar{x} \in D \times R^{m}, \bar{\lambda} \in R^{m}$ satisfying $\phi_{\mathrm{x}}(\overline{\mathrm{x}}, \bar{\lambda})=0$ and $\phi_{\lambda}(\overline{\mathrm{x}}, \bar{\lambda})=0$.

(b) The Hessian matrix $\phi_{x x}(\bar{x}, \bar{\lambda})$ is positive definite.

(c) $\quad \operatorname{rank}(\partial g(\bar{x}) / \partial x)=m$.

Then for any $x \in W(\bar{x})-\{\bar{x}\}$, it holds that

$$
f(\bar{x})<f(x),
$$

where $W(\bar{x})$ is a suitably chosen neighbourhood of $\bar{x}$.

The main theorem in this paper is:

Theorem. Suppose that the same conditions as in lemma 1 are satisfied. 
For arbitrary $x^{(0)} \in R^{n+m}$ and $\lambda^{(0)} \in R^{m}$, define the sequence $\left\{\left(x^{(k)}, \lambda^{(k)}\right) ; k=0\right.$, $1, \ldots\}$ by the following iteration method:

(4) $\left(x^{(k+1)}, \lambda^{(k+1)}\right)$

$$
=\left(x^{(k)}, \lambda^{(k)}\right)-\alpha|| A\left(x^{(k)}, \lambda^{(k)}\right) \|^{-2} p\left(x^{(k)}, \lambda^{(k)}\right) A\left(x^{(k)}, \lambda^{(k)}\right)
$$

$(k=0,1, \ldots)$, where $\alpha$ is a constant such that $0<\alpha<2$ and $\|\cdot\|$ denotes the Euclidean norm.

Then the sequence $\left(x^{(k)}, \lambda^{(k)}\right)$ starting from any initial vectors $x^{(0)} \in U_{0}(\bar{x})$ and $\lambda^{(0)} \in \mathrm{V}_{0}(\bar{\lambda})$ converges to $(\bar{x}, \bar{\lambda})$ given in lemma 1 , as $k$ tends to infinity, where $U_{0}(\bar{x})$ and $V_{0}(\bar{\lambda})$ are suitably chosen neighbourhoods of $\bar{x}$ and $\bar{\lambda}$, respectively.

\section{Preliminaries}

Some preliminaries are required to prove the main theorem. Define $E(x, \lambda)$

as

$$
\begin{aligned}
E(x, \lambda) & =\|p(x, \lambda)\|^{2} \\
& =\left\|\phi_{x}(x, \lambda)\right\|^{2}+\left\|\phi_{\lambda}(x, \lambda)\right\|^{2} .
\end{aligned}
$$

Lemma 2. If $(\bar{x}, \bar{\lambda})$ satisfies condition (a), then it follows that

$$
\operatorname{grad} E(\bar{x}, \bar{\lambda})=0 \text {. }
$$

Proof. From condition (a), it follows that

$$
\begin{aligned}
\partial E(\bar{x}, \bar{\lambda}) / \partial x_{j} & =2 \phi_{x}\left(\left(\partial \phi / \partial x_{j}\right)_{x}\right)^{*}+2 \phi_{\lambda}\left(\left(\partial \phi / \partial x_{j}\right)_{\lambda}\right)^{*} \\
& =0(j=1,2, \ldots, n+m),
\end{aligned}
$$

and

$$
\begin{aligned}
\partial E(\bar{x}, \bar{\lambda}) / \partial \lambda_{i} & =2 \phi_{x}\left(\left(g_{i}\right)_{x}\right)^{*} \\
& =0(i=1,2, \ldots, m) .
\end{aligned}
$$

This proves the lemma.

Lemma 3. Let $G_{j}$ and $H_{i}$ be the Hessian matrices of $\partial \phi / \partial x_{j}(j=1,2, \ldots, n+m)$ and $\partial \phi / \partial \lambda_{i}(i=1,2, \ldots, m)$ respectively. Define an $(n+2 m) \times(n+2 m)$ matrix $c(x, \lambda)$ by

$$
C(x, \lambda)=\sum_{j=1}^{n+m}\left(\partial \phi(x, \lambda) / \partial x_{j}\right) G_{j}+\sum_{i=1}^{m}\left(\partial \phi(x, \lambda) / \partial \lambda_{i}\right) H_{i} \cdot
$$


Then it holds that

$$
\min _{\|\rho\|=1}|| \rho A(\bar{x}, \bar{\lambda})\left\|^{2}>|| C(\bar{x}, \bar{\lambda})\right\| \text {. }
$$

Proof. It follows from conditions (b), (c) and the we11-known fact of matrix rank (see, for example, Beltrami [1, page 144]) that

$$
\operatorname{rank} A(\bar{x}, \bar{\lambda})=n+2 m
$$

Thus

$$
\min _{\|\rho\|=1}|| \rho A(\bar{x}, \bar{\lambda}) \|^{2}>0
$$

On the other hand, condition (a) implies that ||$C(\bar{x}, \bar{\lambda})||=0$. This completes the proof.

4. Proof of the Main Theorem

Denote an $(n+2 m)$-dimensional vector $h^{(k)}$ by

$$
h^{(k)}=\left(x^{(k)}, \lambda^{(k)}\right)-(\bar{x}, \bar{\lambda})
$$

Define $\mu_{k}$ and an $(n+2 m) \times(n+2 m)$ matrix $H(x, \lambda)$ as follows:

and

$$
\mu_{k}=|| A\left(x^{(k)}, \lambda^{(k)}\right) \|^{-2}
$$

$$
H(x, \lambda)=(A(x, \lambda))^{*} A(x, \lambda)+C(x, \lambda) .
$$

Lemma 2 and 3 are useful in proving the main theorem in a similar way to Yamamoto [8]. Since

$$
\operatorname{grad} E(x, \lambda)=2 p(x, \lambda) A(x, \lambda),
$$

it follows from (4) and lemma 2 that the recurrence relations

$$
h^{(k+1)}=h^{(k)}-\frac{1}{2} \alpha \mu_{k}\left\{\operatorname{grad} E\left(x^{(k)}, \lambda^{(k)}\right)-\operatorname{grad} E(\bar{x}, \bar{\lambda})\right\}
$$

hold for $k=0,1, \ldots$. As is easily shown, $2 H(x, \lambda)$ is the Hessian matrix of $E(x, \lambda)$. By Taylor's expansion, (5) is rewritten as the following form:

$$
\begin{aligned}
h^{(k+1)} & =h^{(k)}-\alpha \mu_{k} h^{(k)} \int_{0}^{1} H\left(\bar{x}+t\left(x^{(k)}-\bar{x}\right), \bar{\lambda}+t\left(\lambda^{(k)}-\bar{\lambda}\right)\right) d t \\
& =h^{(k)}\left(I-J_{k}\right),
\end{aligned}
$$

where $I$ is the identity matrix and

$$
J_{k}=\alpha \mu_{k} \int_{0}^{1} H\left(\bar{x}+t\left(x^{(k)}-\bar{x}\right), \bar{\lambda}+t\left(\lambda^{(k)}-\bar{\lambda}\right)\right) d t .
$$


Let $\varepsilon$ be an arbitrarily fixed positive constant such that

$$
\cdot 0<\varepsilon<\min \{d, e(\alpha)\} \text {, }
$$

where

and

$$
d \equiv \min _{\|\rho\|=1}\|\rho A(\bar{x}, \bar{\lambda})\|^{2}
$$

$$
e(\alpha)=\frac{2-\alpha}{2 \alpha+2}\|A(\bar{x}, \bar{\lambda})\|^{2} \text {. }
$$

Then lemma 3 implies that $d>0$ and $e(\alpha)>0$ for constant $\alpha$ such as $0<\alpha<2$. Thus for any $\varepsilon$ satisfying (7), there exists a positive constant $\delta$ such that

$$
z \in \mathrm{U}_{0}(\overline{\mathrm{x}}) \times \mathrm{V}_{0}(\bar{\lambda}) \equiv\{(\mathrm{x}, \lambda) ;\|(\mathrm{x}, \lambda)-(\overline{\mathrm{x}}, \bar{\lambda})\|<\delta\}
$$

implies

$$
\begin{gathered}
\|C(z)\| \leq \varepsilon \\
\|A(\bar{x}, \bar{\lambda})\|^{2}-\varepsilon \leqq\|A(z)\|^{2} \leqq\|A(\bar{x}, \bar{\lambda})\|^{2}+\varepsilon
\end{gathered}
$$

and

$$
\mathrm{d}-\varepsilon \leqq \min _{\|\rho\|=1} \rho \mathrm{H}(\mathrm{z}) \rho^{*} .
$$

Then for $\left(\mathrm{x}^{(\mathrm{k})}, \lambda^{(\mathrm{k})}\right) \in \mathrm{U}_{0}(\overline{\mathrm{x}}) \times \mathrm{V}_{0}(\bar{\lambda})$,

$$
\mu \equiv \frac{\alpha(\mathrm{d}-\varepsilon)}{\|\mathrm{A}(\overline{\mathrm{x}}, \bar{\lambda})\|^{2}+\varepsilon} \leqq \rho J_{\mathrm{k}} \rho^{*} \leqq \frac{\alpha\left(\|\mathrm{A}(\overline{\mathrm{x}}, \bar{\lambda})\|^{2}+2 \varepsilon\right)}{\|\mathrm{A}(\overline{\mathrm{x}}, \bar{\lambda})\|^{2}-\varepsilon} \equiv \mathrm{M},
$$

and $0<\mu<M<2$. Let $K=\max (|1-\mu|,|1-M|)$. Then it holds that $0<K<1$. Clearly

$$
1-\mathrm{M} \leqq \rho\left(I-\mathrm{J}_{\mathrm{k}}\right) \rho^{*} \leqq 1-\mu .
$$

Since $I-J_{k}$ is a symmetric matrix, it follows from a result of the matrix theory (see, for example, Varga [7, page 11]) that

$$
\max _{\|\rho\|=1}\left\|\left(I-J_{k}\right) \rho^{*}\right\|=\max _{\|\rho\|=1}\left|\rho\left(I-J_{k}\right) \rho^{*}\right| \text {. }
$$

By (8) and (9),

$$
\text { (10) } \max _{\|\rho\|=1}\left\|\rho\left(I-J_{k}\right)\right\| \leq \mathrm{K}
$$

Let $\xi^{(k)}=h^{(k)} /\left\|h^{(k)}\right\|$. Then, from (6) and (10), it holds that 


$$
\begin{aligned}
\left\|h^{(k+1)}\right\| & =\left\|h^{(k)}\right\|\left\|\xi^{(k)}\left(I-J_{k}\right)\right\| \\
& \left.\leqq\left\|h^{(k)}\right\| \max _{\|\rho\|=1}\left\|\rho\left(I-J_{k}\right)\right\|\right) \\
& \leqq K\left\|h^{(k)}\right\| .
\end{aligned}
$$

This completes the proof of the main theorem.

5. Numerical Example

As a numerical example, let us consider the following Rosen-Suzuki Test Problem [6]:

Minimize

$$
f=x_{1}^{2}+x_{2}^{2}+2 x_{3}^{2}+x_{4}^{2}-5 x_{1}-5 x_{2}-21 x_{3}+7 x_{4}
$$

subject to

$$
\begin{aligned}
& \mathrm{x}_{1}{ }^{2}+\mathrm{x}_{2}{ }^{2}+\mathrm{x}_{3}{ }^{2}+\mathrm{x}_{4}{ }^{2}+\mathrm{x}_{1}-\mathrm{x}_{2}+\mathrm{x}_{3}-\mathrm{x}_{4}-8 \leqq 0, \\
& \mathrm{x}_{1}{ }^{2}+2 \mathrm{x}_{2}{ }^{2}+\mathrm{x}_{3}{ }^{2}+2 \mathrm{x}_{4}{ }^{2}-\mathrm{x}_{1} \quad-\mathrm{x}_{4}-10 \leqq 0, \\
& 2 \mathrm{x}_{1}{ }^{2}+\mathrm{x}_{2}{ }^{2}+\mathrm{x}_{3}{ }^{2}+2 \mathrm{x}_{1}-\mathrm{x}_{2}-\mathrm{x}_{4}-5 \leqq 0 .
\end{aligned}
$$

Minimum point and corresponding value of $f$ are given by

$$
\left(\bar{x}_{1}, \bar{x}_{2}, \bar{x}_{3}, \bar{x}_{4}\right)=(0,1,2,-1)
$$

and

$$
f\left(\bar{x}_{1}, \bar{x}_{2}, \bar{x}_{3}, \bar{x}_{4}\right)=-44 \text {. }
$$

Let us stop the iteration process (4) if

$$
\left|x_{j}(k+1)-x_{j}^{(k)}\right|<\varepsilon(j=1,2, \ldots, 7)
$$

and

$$
\left|\lambda_{i}{ }^{(k+1)}-\lambda_{i}{ }^{(k)}\right|<\varepsilon(i=1,2,3)
$$

are satisfied, where $\varepsilon$ is a suitably chosen positive constant. The above problem can be also solved by SUMT transformation and Davidon-Fletcher-Powell method [2], [3]. The numerical computations with $\varepsilon=10^{-4}$ were carried out on a FACOM 230-75 computer of Kyoto University Computation Center. These results 
are shown in the following table.

Table. Numerical Solutions for Rosen-Suzuki Problem

Present method

Initial data $(0.0,0.0, \ldots, 0.0)$
SUMT transformation

$(0.0,0.0,0.0,0.0)$

\begin{tabular}{r|rrrr} 
& $\alpha=0.9$ & 1.3 & 1.9 & \\
\hline $\mathrm{x}_{1}$ & -0.04145 & -0.04222 & -0.04024 & 0.00735 \\
$\mathrm{x}_{2}$ & 1.16725 & 1.17805 & 1.09902 & 1.00012 \\
$\mathrm{x}_{3}$ & 1.94409 & 1.94038 & 1.98684 & 1.99927 \\
$\mathrm{x}_{4}$ & -1.07554 & -1.07123 & -1.05832 & -1.00726 \\
$\mathrm{f}$ & -43.9042 & -43.8589 & -43.9141 & -43.9983 \\
\hline $\begin{array}{c}\text { CPU TIME } \\
(\mathrm{sec})\end{array}$ & 3.9 & 3.3 & 2.4 & 2.6
\end{tabular}

\begin{tabular}{c|cccc} 
& $\alpha=0.9$ & 1.3 & 1.9 & \\
\hline $\mathrm{x}_{1}$ & 0.01438 & 0.01254 & 0.00207 & 0.00104 \\
$\mathrm{x}_{2}$ & 0.99685 & 0.99625 & 0.99337 & 0.99886 \\
$\mathrm{x}_{3}$ & 2.01153 & 2.00789 & 2.00202 & 1.99924 \\
$\mathrm{x}_{4}$ & -0.97818 & -0.98709 & -0.99339 & -1.00085 \\
$\mathrm{f}$ & -44.0120 & -44.0076 & -43.9834 & -43.9927 \\
\hline CPU TIME & 3.7 & 3.2 & 2.6 & 5.8 \\
$(\mathrm{sec})$ & & &
\end{tabular}

Initial data $(1.1,1.1, \ldots, 1.1) \quad(1.1,1.1,1.1,1.1)$

\begin{tabular}{c|cccc} 
& $\alpha=0.9$ & 1.3 & 1.9 & \\
\hline $\mathrm{x}_{1}$ & 0.00111 & 0.00643 & -0.00213 & 0.00124 \\
$\mathrm{x}_{2}$ & 0.99227 & 0.99246 & 0.99004 & 0.99902 \\
$\mathrm{x}_{3}$ & 2.00754 & 2.00481 & 1.99968 & 1.99897 \\
$\mathrm{x}_{4}$ & -0.97278 & -0.98812 & -1.00219 & -1.00114 \\
$\mathrm{f}$ & -43.9933 & -43.9875 & -43.9661 & -43.9982 \\
\hline $\begin{array}{c}\text { CPU TIME } \\
(\text { sec })\end{array}$ & 3.9 & 3.5 & 2.9 & 48.0
\end{tabular}




\section{Remark}

The present iteration method (4) is based on the previous method [5], minimizing a sum of squares of nonlinear functions. The present method is simple in the sense that it need not compute the inverse of the Hessian matrix. Moreover, the computation results in $\$ 5$ show that the present method is rather better than SUMT transformation, so far as computation time is concerned. It should be noted that the present method requires more storage capacity than SUMT transformation.

\section{References}

[1] Beltrami, E.J., An Algorithmic Approach to Nonlinear Analysis and Optimization, Academic Press, New York and London, 1970.

[2] Fiacco, A.V. and G.P.McCormic, "Computational Algorithm for the Sequential Unconstrained Minimization Technique for Nonlinear Programming," Management Sci., 10(1964), 601-617.

[3] Fletcher, R. and M.J.D.Powe11, "A Rapidly Convergent Descent Method for Minimization," Computer J., 6(1963), 163-168.

[4] Hadley, G., Nonlinear and Dynamic Programming, Addison-Wesley, Reading, Mass., 1964.

[5] Noda, T., "On an Iteration Method for Finding a Local Minimum of $\sum_{i=1}^{m}\left(f_{i}\left(x_{1}, x_{2}, \ldots, x_{n}\right)\right)^{2}$ (in Japanese)," Sügaku (Edited by the Mathematical Society of Japan), 26(1974), 37-40.

[6] Rosen, J.B. and S.Suzuki, "Construction of Nonlinear Programming Test Problems," Comm. ACM, 8(1965), 113.

[7] Varga, R.S., Matrix Iterative Analysis, Prentice-Ha11, Englewood Cliffs, New Jersey, 1962.

[8] Yamamoto, T., "On Noda Method for Finding a Local Minimum of 
$\sum_{i=1}^{m}\left(f_{i}\left(x_{1}, x_{2}, \ldots, x_{n}\right)\right)^{2}$ (in Japanese)," Sügaku (Edited by the Mathematical Society of Japan), 26(1974), 349-351. 